\title{
XMM-Newton observations of the Small Magellanic Cloud: Long term evolution of frequently observed Be/X-ray binaries ${ }^{\star}$
}

\author{
P. Eger and F. Haberl
}

\begin{abstract}
Max-Planck-Institut für extraterrestrische Physik, Giessenbachstraße, 85748 Garching, Germany
e-mail: peger@mpe.mpg.de
\end{abstract}

Received 15 August 2008 / Accepted 9 September 2008

\begin{abstract}
Aims. To follow the long term evolution of Be/X-ray binaries in the Small Magellanic Cloud (SMC) we analyzed twenty-two XMM-Newton observations towards the calibration target 1E 0102.2-7218 and seven observations towards the neighboring region around the eclipsing binary HD 5980.

Methods. The data from the European Photon Imaging Cameras (EPIC) were used to produce images, X-ray spectra and light curves in different energy bands. We performed a detailed spectral and temporal analysis on the data of all known Be/X-ray binary pulsars that have been detected with a sufficiently high number of counts. Fast Fourier Transformation (FFT) power spectra were produced to search for X-ray pulsations and to determine their significance. To derive accurate values for the pulse periods we utilized the Bayesian method. To identify or confirm the optical counterparts we derived the X-ray positions of the pulsars using maximum likelihood source detection techniques and correlated them with optical catalogues.

Results. There are ten known Be/X-ray binaries located in the observed fields. In addition to the previously published results for three of these sources, we present long term X-ray light curves and pulse period histories for the following six pulsars: CXOU J005736.2721934 (565 s), RX J0057.8-7207 (152 s), RX J0059.3-7223 (201 s), CXOU J010102.7-720658 (304 s), RX J0101.3-7211 (455 s), SAX J0103.2-7209 (345 s) and RX J0103.6-7201 (1323 s). We do not agree with the identification of the ROSAT source RX J0105.1$7211=$ XMMU J010509.7-721146 as the 3.34 s ASCA pulsar AXJ0105-722 and suggest that RX J0105.1-7211 is more likely a background AGN. We find soft excess emission around $1 \mathrm{keV}$ in the X-ray spectra of two pulsars (SAX J0103.2-7209 and RX J0103.67201). The spin history in combination with the long term X-ray light-curve indicate a type II outburst of CXOU J010102.7-720658 at the end of 2005 and a reversal in the accretion disc torque in SAX J0103.2-7209 in May 2002. An X-ray ephemeris for the binary orbit was only available in the literature for CXOU J005736.2-721934 and is in good agreement with the observed XMM-Newton light-curve.
\end{abstract}

Key words. galaxies: individual: Small Magellanic Cloud - galaxies: stellar content - stars: emission-line, Be - stars: neutron $\mathrm{X}$-rays: binaries

\section{Introduction}

Almost every year new Be/X-ray binary pulsars are discovered in the Small Magellanic Cloud (SMC). To date, there are $\sim 50$ pulsars with Be star companions known in this nearby dwarf galaxy (for recent work on XMM-Newton see, e.g., Haberl et al. 2008a). To explore the nature and evolution of these kinds of systems collecting a large sample of pulsars is as important as the monitoring of single systems over longer periods of time.

High mass X-ray binaries are highly variable sources and can show a dramatic increase in brightness during outburst. Pulsars with Be companion especially show recurrent outbursts (type I) when the neutron star approaches the circumstellar disc during periastron passage which significantly enhances the mass accretion rate. Longer and even brighter outbursts (type II) are seen when the massive Be star repels its disc and large amounts of matter cross the neutron star orbit.

With the large field of view (FoV) of the Rossi X-ray timing explorer (RXTE) all pulsars in the SMC can be observed on a regular basis (for recent results see Galache et al. 2008). However, to locate the sources, identify the optical counterpart

* Based on observations with XMM-Newton, an ESA Science Mission with instruments and contributions directly funded by ESA Member states and the USA (NASA). and to derive proper spectral parameters it is necessary to detect the pulsars with imaging telescopes like Chandra and XMM-Newton.

Most regions in the SMC have only been covered once or twice with the EPIC instruments on XMM-Newton. However, the two adjacent fields, around the calibration source 1E0102.27218 and around the eclipsing binary HD 5980, in the northeastern part of the SMC have been frequently observed with XMM-Newton (twenty-two times and seven times, respectively). Since there are several Be/X-ray binaries located in this region, these observations can be used to study the long term evolution of this sample of pulsars over the last 7.5 years.

The results for three of the Be/X-ray binaries in the northern part of the SMC have already been published: Haberl \& Pietsch (2007) reported about all XMM-Newton detections of the $280 \mathrm{~s}$ ASCA pulsar RX J0057.8-7202, and Eger \& Haberl (2008) discovered pulsations with a period of $\sim 726 \mathrm{~s}$ from RX J0105.97203 which is located south-east of the SNR 1E0102.2-7218. Moreover, Haberl et al. (2008a) detected the $201 \mathrm{~s}$ pulsar RX J0059.3-7223 with a recent XMM-Newton observation.

In this paper we report about the long term evolution of all other known pulsars in this region detected with XMM-Newton. 
Table 1. Details of the XMM-Newton EPIC observations.

\begin{tabular}{|c|c|c|c|c|c|}
\hline $\begin{array}{l}\text { Observation } \\
\text { ID }\end{array}$ & Start time & End time & \multicolumn{3}{|c|}{$\begin{array}{c}\text { Exposures }^{1} \\
\text { noScr/Spec/Timing }\end{array}$} \\
\hline 0123110201 & 2000-04-16 19:06:50 & 2000-04-17 01:27:02 & 19298 / 18433 / 17727 & 18794 / 17727 / 17727 & 18794 / 17727 / 17727 \\
\hline 0123110301 & 2000-04-17 03:41:01 & 2000-04-17 09:44:33 & $18298 / 12343 / 11560$ & 17794 / 11565 / 11560 & 17794 / $11573 / 11560$ \\
\hline 0110000201 & 2000-10-17 14:56:47 & $2000-10-17$ 20:49:36 & 15848 / $12350 / 11599$ & 19744 / 12300 / 11599 & 19744 / 12300 / 11599 \\
\hline 0135720601 & 2001-04-14 20:35:50 & 2001-04-15 06:00:17 & $16198 / 9500 / 9500$ & 32894 / 26134 / 9500 & 32894 / 26126 / 9500 \\
\hline 0018540101 & 2001-11-20 23:35:02 & 2001-11-21 07:18:00 & 24408 / 20876 / 20234 & 27022 / 20958 / 20234 & 27022 / 20956 / 20234 \\
\hline 0135720801 & 2001-12-25 17:58:49 & 2001-12-26 03:44:30 & 31310 / 30574 / 28420 & 32127 / 29378 / 28420 & 32127 / 29394 / 28420 \\
\hline 0084200101 & 2002-03-30 13:36:46 & 2002-03-30 19:47:18 & $18998 / 14573 / 17761^{2}$ & $21274 / 14573 / 17761^{2}$ & $21274 / 14571 / 17761^{2}$ \\
\hline 0135721001 & 2002-05-18 10:15:27 & 2002-05-18 19:45:51 & 12214 / 10871 / 10258 & 16684 / 10268 / 10258 & 14884 / 10257 / 10258 \\
\hline 0135721101 & 2002-10-13 03:01:34 & $2002-10-13$ 09:58:53 & 10233 / 9837 / 9836 & 23672 / 23269 / 23268 & 23672 / $23271 / 23268$ \\
\hline 0135721301 & 2002-12-14 03:35:52 & 2002-12-14 11:55:52 & 14538 / 14219 / 14195 & 28672 / 28318 / 28274 & 28672 / $28310 / 28274$ \\
\hline 0135721401 & 2003-04-20 11:46:51 & 2003-04-21 00:42:41 & 18735 / 18724 / 18724 & 34530 / 34498 / 34492 & 34529 / 34504 / 34492 \\
\hline 0135721501 & 2003-10-27 07:37:01 & 2003-10-27 16:22:31 & 28534 / 26948 / 26094 & 30172 / 26965 / 26094 & 30177 / 26995 / 26094 \\
\hline 0135721701 & $2003-11-16$ 05:54:00 & $2003-11-16$ 15:03:20 & 25975 / 25549 / 24834 & 27300 / 24859 / 24834 & $27300 / 24900 / 24834$ \\
\hline 0135721901 & 2004-04-28 06:51:55 & 2004-04-28 16:28:37 & 31798 / 31740 / 31745 & 33274 / 31617 / 31745 & 33279 / 31639 / 31745 \\
\hline 0135722401 & 2004-10-14 08:45:59 & 2004-10-14 7:42:39 & 30664 / 30580 / 30371 & 30872 / 30454 / 30371 & 30877 / 30470 / 30371 \\
\hline 0135722001 & 2004-10-26 06:38:37 & 2004-10-26 15:48:37 & 29657 / 29574 / 29526 & 31672 / 29720 / 29526 & 31677 / 29720 / 29526 \\
\hline 0135722101 & 2004-11-06 22:20:01 & 2004-11-06 12:28:21 & 29935 / 22722 / 22710 & 31572 / 22784 / 22710 & 31577 / 22778 / 22710 \\
\hline 0135722201 & not used due to high $\mathrm{b}$ & ackground & & & \\
\hline 0135722301 & 2004-11-07 22:17:44 & 2004-11-08 07:27:07 & 29998 / 11215 / 11172 & 31635 / 21496 / 11172 & $31640 / 21493 / 11172$ \\
\hline 0135722501 & 2005-04-17 21:57:56 & 2005-04-18 08:34:36 & $34870 / 21802$ / 21783 & 36872 / 21927 / 21783 & 36877 / 21932 / 21783 \\
\hline 0135722601 & 2005-11-05 06:27:01 & 2005-11-05 15:12:30 & 29993 / 29939 / 29186 & 30201 / 29525 / 29186 & 30206 / 29527 / 29186 \\
\hline 0304250401 & 2005-11-27 06:04:36 & 2005-11-27 11:19:36 & 15935 / 15865 / 15829 & 17567 / 17485 / 15829 & 17572 / 17493 / 15829 \\
\hline 0304250501 & 2005-11-29 04:53:24 & 2005-11-29 09:51:44 & 14935 / 14932 / 14932 & 16567 / 16564 / 16564 & 16572 / 16569 / 16564 \\
\hline 0304250601 & 2005-12-11 12:30:32 & 2005-12-11 17:30:32 & 10565 / 10565 / 10565 & 16667 / 16663 / 10565 & 16672 / 16668 / 10565 \\
\hline 0135722701 & 2006-04-20 02:07:01 & 2006-04-20 10:52:31 & 29993 / 29991 / 29869 & 30201 / 30201 / 30201 & 30206 / 30203 / 30201 \\
\hline 0412980101 & 2006-11-05 00:37:16 & 2006-11-05 09:55:36 & 31964 / 31933 / 30814 & 32172 / 30996 / 30814 & 32177 / 31009 / 30814 \\
\hline 0412980201 & 2007-04-25 12:18:24 & $2007-04-25$ 22:43:24 & 35964 / 35237 / 22794 & 36172 / 22904 / 22794 & 36177 / 22914 / 22794 \\
\hline 0500980201 & 2007-06-06 08:34:03 & 2007-06-06 16:54:03 & 27035 / 19821 / 19796 & 28667 / 21400 / 19796 & 28672 / 21397 / 19796 \\
\hline 0412980301 & 2007-10-26 09:31:06 & 2007-10-26 20:07:46 & 36664 / 36663 / 33833 & 36872 / 34167 / 33833 & 36877 / 34170 / 33833 \\
\hline
\end{tabular}

${ }^{1}$ Exposure time without background screening/net exposure time with background screening used for spectra/net exposure time within the common PN/MOS GTI used for timing analysis. In the case of PN small window observations the latter value for PN gives the common interval of all three instruments, whereas the MOS value is the common GTI of the MOS cameras only. ${ }^{2}$ Timing analysis without background screening, to obtain a reasonably long common exposure interval.

\section{Data analysis}

The set of twenty-nine XMM-Newton observations which we used for our monitoring is summarized in Table 1. For the X-ray analysis we used the XMM-Newton Science Analysis System (SAS) version 7.1.0, tools from the FTOOLS package for part of the timing analysis and XSPEC version 11.3.2p for spectral modelling.

We determined the position of the sources using the SAS standard maximum likelihood technique for source detection and performed an astrometric bore-sight correction afterwards (see also Haberl et al. 2008b). A background screening was applied for source detection and spectral and timing analyses.

We searched for X-ray pulsations in the EPIC data for all sources with at least a total of 200 source counts in the $0.2-4.5 \mathrm{keV}$ band in the three instruments. After correcting the photon arrival times to the solar system barycenter, we combined the EPIC-PN (Strüder et al. 2001) and MOS (Turner et al. 2001) data and accepted only those events within the common good time interval (GTI) of all three cameras (considering both instrument GTI and background screening). The resulting net exposures are listed in Table 1 . In some observations the PN camera was operated in small window mode. In these cases, when a source was outside of the PN but still inside the MOS FoV, we used the common MOS1 and MOS2 GTI (see footnote ${ }^{1}$ to Table 1). To derive accurate values and errors for the pulsation period we used the Bayesian method (Gregory \& Loredo 1996) as described in Zavlin et al. (2000).

For the analysis of the X-ray spectra we extracted pulsephase averaged EPIC spectra for PN (single + double pixel events, PATTERN 0-4) and MOS (PATTERN 0-12) disregarding bad CCD pixels and columns (FLAG 0). Only those detections with at least 200 source counts from the source detection analysis in the $0.2-4.5 \mathrm{keV}$ band were considered. For each source the EPIC spectra from the same observation were simultaneously fit with an absorbed powerlaw model only allowing for a normalization factor between the spectra. We used two absorption components, accounting for the Galactic foreground absorption (with a fixed hydrogen column density of $6 \times 10^{20} \mathrm{~cm}^{-2}$ and elemental abundances from Wilms et al. 2000) and the SMC absorption (with column density as a free parameter in the fit and with metal abundances reduced to 0.2 as typical for the SMC; Russell \& Dopita 1992).

In this paper we give intrinsic source luminosities that we derived from the observed flux with the absorption set to zero for an SMC distance of $60 \mathrm{kpc}$ (Hilditch et al. 2005). Luminosities taken from the literature have been scaled to the same distance, when necessary.

We note here that fluxes and luminosities given by Sasaki et al. (2003) were not vignetting and PSF corrected, which leads to systematically underestimated values, in particular for sources at large off-axis angles. 


\section{Results and discussion}

In the following, we present long term X-ray light curves and pulse period histories for seven pulsars: CXOU J005736.2721934 (565 s), RX J0057.8-7207 (152 s), RX J0059.3-7223 (201 s; see also Haberl et al. 2008a), CXOU J010102.7720658 (304 s), RX J0101.3-7211 (455 s), SAX J0103.2-7209 (345 s) and RXJ0103.6-7201 (1323 s). Moreover, we investigate the case of the ROSAT source RXJ0105.1-7211 = XMMU J010509.7-721146 which turned out to be misidentified with the 3.34 s ASCA pulsar AX J0105-722. The Be/XRB candidate RX J0101.6-7204 is also located in the northern part of the SMC, however, the XMM-Newton detections suggest that this source is rather a background AGN.

\subsection{The 565 s Be/X-ray binary pulsar CXOU J005736.2-721934}

History: The pulse period of $(564.81 \pm 0.41) \mathrm{s}$ for this $\mathrm{Be} / \mathrm{X}$-ray binary has only been measured with Chandra so far during a $100 \mathrm{ks}$ observation in May 2001 (Macomb et al. $2003)$ at a luminosity of $6.2 \times 10^{34} \mathrm{erg} \mathrm{s}^{-1}(0.6-7.5 \mathrm{keV}$, unabsorbed). Sasaki et al. (2003) detected CXOU J005736.2721934 in two XMM-Newton observations (0110000201: $L_{\mathrm{X}}=$ $\left.0.82 \times 10^{34} \mathrm{erg} \mathrm{s}^{-1}, 0084200101: L_{\mathrm{x}}=4.09 \times 10^{34} \mathrm{erg} \mathrm{s}^{-1}\right)$, but were not able to confirm this long period. Furthermore, Nazé et al. (2004) reported about a relatively bright XMM-Newton detection $\left(6.93 \times 10^{35} \mathrm{erg} \mathrm{s}^{-1}\right)$ in the $27 \mathrm{ks}$ observation 0018540101 , but they were also not able to find any periodicity.

New results: In addition to the three XMM-Newton detections mentioned above, the source was weakly detected another four times in this survey. We reexamined the data of the only bright detection (0018540101), but were also not able to find pulsations.

Figure 1 shows the long term X-ray light-curve including all observations. For the fainter detections where no spectral analysis was possible, we give luminosities derived from count-rates by assuming the spectral form of the brightest detection. Due to the large off-axis angle we do not give luminosities from Sasaki et al. (2003), (see Sect. 2).

In accordance with the X-ray ephemeris from Galache et al. (2008), CXOU J005736.2-721934 was only bright during those observations when the source was near to the proposed periastron passage (obs. 0018540101 with phase $0.10 \pm 0.09$ and obs. 0084200101 with phase $0.95 \pm 0.10$ ).

\subsection{The 152 s Be/X-ray binary pulsar $R X J 0057.8-7207$}

History. Pulsations with a period of $152.098 \pm 0.016 \mathrm{~s}$ from this ROSAT classified candidate (Haberl \& Sasaki 2000) have been detected with Chandra by Macomb et al. (2003). Independently, Sasaki et al. (2003) measured a period of $152.34 \pm 0.05 \mathrm{~s}$ in the XMM-Newton observation 0110000201.

New results. We detected this source in another four observations, but due to the continuously decreasing source luminosity we were not able to determine the period in any of them. A spectrum could still be produced for observation 0018540101, whereas the luminosities for all following observations were determined from the count-rate by assuming the same spectral

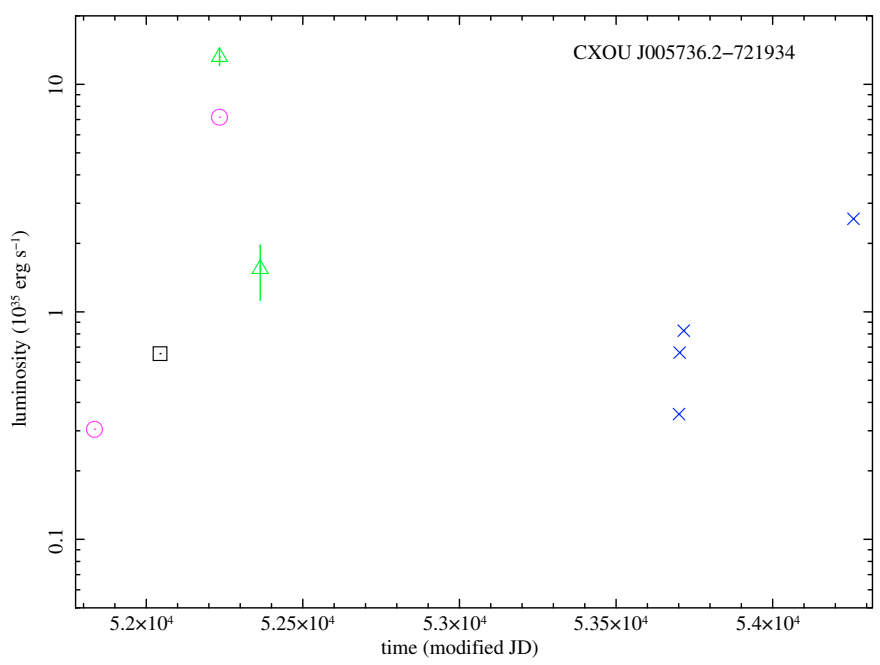

Fig. 1. Long term X-ray light-curve of CXOU J005736.2-721934. Square (black): Macomb et al. (2003) (Chandra, 0.6-7.5 keV, unabsorbed), circles (magenta): Nazé et al. (2004) (XMM-Newton, 0.4-10.0 keV, absorbed), triangles (green): this work (from spectrum, $0.2-10.0 \mathrm{keV}$, unabsorbed), crosses (blue): this work (from count-rate, $0.2-10.0 \mathrm{keV}$, unabsorbed).

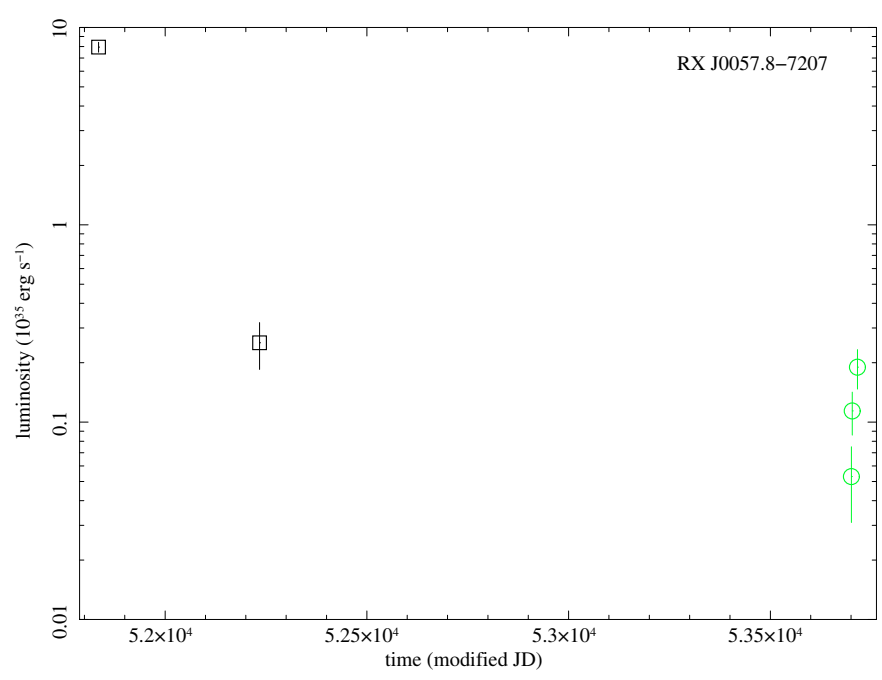

Fig. 2. Long term X-ray light-curve of RX J0057.8-7207. Squares (black): luminosities from spectral modelling, circles (green): luminosities from count-rate by assuming the same spectral form.

form. We re-determined the flux for observation 0110000201 and show the long term X-ray light-curve in Fig. 2.

Since there is no X-ray ephemeris available for this pulsar, it is not clear whether the changes in the X-ray luminosity are correlated to the orbital phase.

\subsection{The 201 s Be/X-ray binary pulsar RX J0059.3-7223}

History. This ROSAT source was classified as an XRB candidate by Kahabka et al. (1999) using hardness ratio criteria. Sasaki et al. (2003) detected it with XMM-Newton in the observations 0110000201 and 0084200101 . Majid et al. (2004) found pulsations, strongest at energies above $2 \mathrm{keV}$, with a period of $201.9 \pm 0.5 \mathrm{~s}$ in the data from observation 0018540101 . They also found evidence for this periodicity in an XMM-Newton observation 529 days later. 


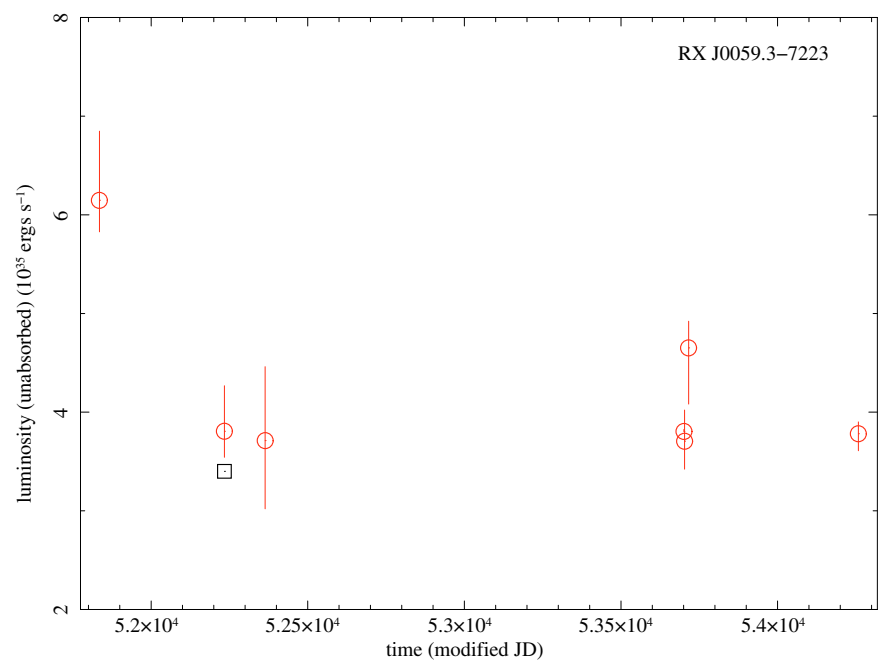

Fig.3. Long term X-ray light-curve of RXJ0059.3-7223. Square (black): Majid et al. (2004) (XMM-Newton, 0.2-6.0 keV), circles (red): this work.

New results. We detected RXJ0059.3-7223 in four more XMM-Newton observations, but we were only able to confirm the period in observation 0500980201. Spectra, timing analyses and pulse profiles recently have been published (Haberl et al. 2008a). Apart from the first detection, RX J0059.3-7223 is seen at a rather constant brightness of $\sim 3.8 \times 10^{35} \mathrm{erg} \mathrm{s}^{-1}$ (see Fig. 3).

\subsection{The $304 \mathrm{~s} B$ Be/X-ray binary pulsar CXOU J010102.7-720658}

History. Pulsations with a period of $304.49 \pm 0.13 \mathrm{~s}$ were first discovered in a $100 \mathrm{ks}$ Chandra observation in May 2001 (Macomb et al. 2003). Later Sasaki et al. (2003) and Nazé et al. (2004) detected this source in the XMM-Newton observations 0110000201, 0135720601 and 0018540101, but were not able to confirm the period, due to the low count-rate.

New results. CXOU J010102.7-720658 was in the field of view of sixteen XMM-Newton observations and was detected in fifteen. In five cases the source was bright enough to perform spectral and timing analysis and we were able to confirm the pulsations in all of them. The pulse profile (Fig. 4, left) consists of a double peaked main pulse with an energy dependent shape.

Figure 5 shows the long term luminosity and pulse period history. CXOU J010102.7-720658 appears at luminosities below $2 \times 10^{35} \mathrm{erg} \mathrm{s}^{-1}$ with one exception in November 2005 where the luminosity increased by a factor of $\sim 6$. After this event the pulsar showed strong spin-up, in contrast to a period of slight spindown (0.13 s/year) during the lower luminosity states before. Since there is no X-ray ephemeris available in the literature for this source, it is not clear whether the changes in brightness are correlated to the orbital phase.

However, the coincidence of the increase in the X-ray luminosity and the sudden spin-up may indicate a type II outburst in November 2005 that we only observed during its declining phase.

\subsection{The 455 s Be/X-ray binary pulsar RX J0101.3-7211}

History. Sasaki et al. (2001) discovered $455 \pm 2$ s pulsations with XMM-Newton in observation 0123110201 and confirmed
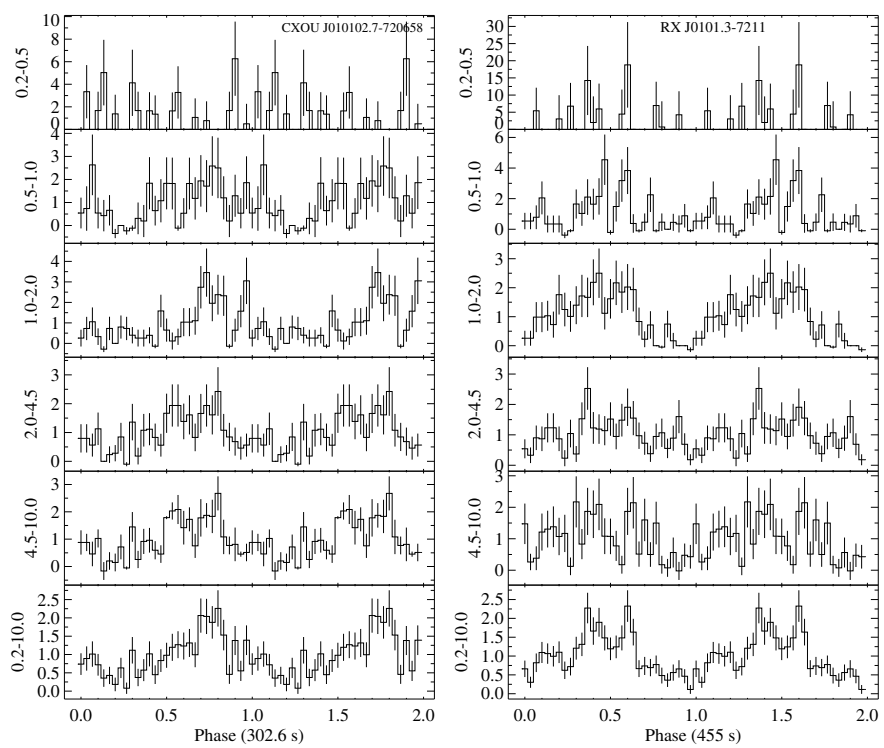

Fig. 4. Left: pulse profile of CXOU J010102.7-720658 from observation 0304250401, right: pulse profile of RX J0101.3-7211 from observation 0123110201 .

this period by examining ROSAT archival data between 1993 and 1998. Additionally, they report a brightness variability between $0.5 \times 10^{35} \mathrm{erg} \mathrm{s}^{-1}$ and $2.5 \times 10^{35} \mathrm{erg} \mathrm{s}^{-1}$ in the ROSAT band (0.1-2.4 keV) between the years 1993 and 2000. Using the newer XMM-Newton data from observation 0110000201, Sasaki et al. (2003) detected a shorter period of $452.2 \pm 0.5 \mathrm{~s}$.

New results. Including the XMM-Newton observations mentioned above, RX J0101.3-7211 was in the FoV of eight pointings. The data were of sufficient statistical quality to perform spectral and temporal analyses, and we detected significant pulsations in six observations (see Fig. 6, bottom).

Although the measured pulse periods vary from $447 \mathrm{~s}$ to $454.5 \mathrm{~s}$, they are all, with one exception, consistent within $2 \sigma$ errors. The scatter might be caused by long term brightness variations of the source during the observation that influence the timing analysis for longer pulse periods as in this case. The pulse profile consists of a broad, double peaked main pulse (see Fig. 4, right).

Furthermore, we can confirm the variability of this source reported by Sasaki et al. (2001) by observing luminosities between $1 \times 10^{35} \mathrm{erg} \mathrm{s}^{-1}$ and $6 \times 10^{35} \mathrm{erg} \mathrm{s}^{-1}$ (see Fig. 6, top). The light-curve might indicate a period of higher activity in 2000 with gradual fading afterwards. In this case the spin-up during this period could be real.

Unfortunately, there is no X-ray ephemeris available in the literature for this source, and it is therefore not clear whether the changes in the X-ray luminosity are correlated to the orbital phase.

\subsection{The Be/X-ray binary candidate $R X$ J0101.6-7204}

History. The ROSAT source RX J0101.6-7204 was classified as $\mathrm{Be} / \mathrm{XRB}$ candidate due to its correlation with the emission line star MA93 1277 (Haberl \& Sasaki 2000). This source was first detected with XMM-Newton by Sasaki et al. (2003) (obs.: 0110000201 and 0135720601) and by Nazé et al. (2004) (obs.: 0110000201 and 0018540101). The X-ray spectra of all XMM-Newton detections could be modelled with an absorbed 

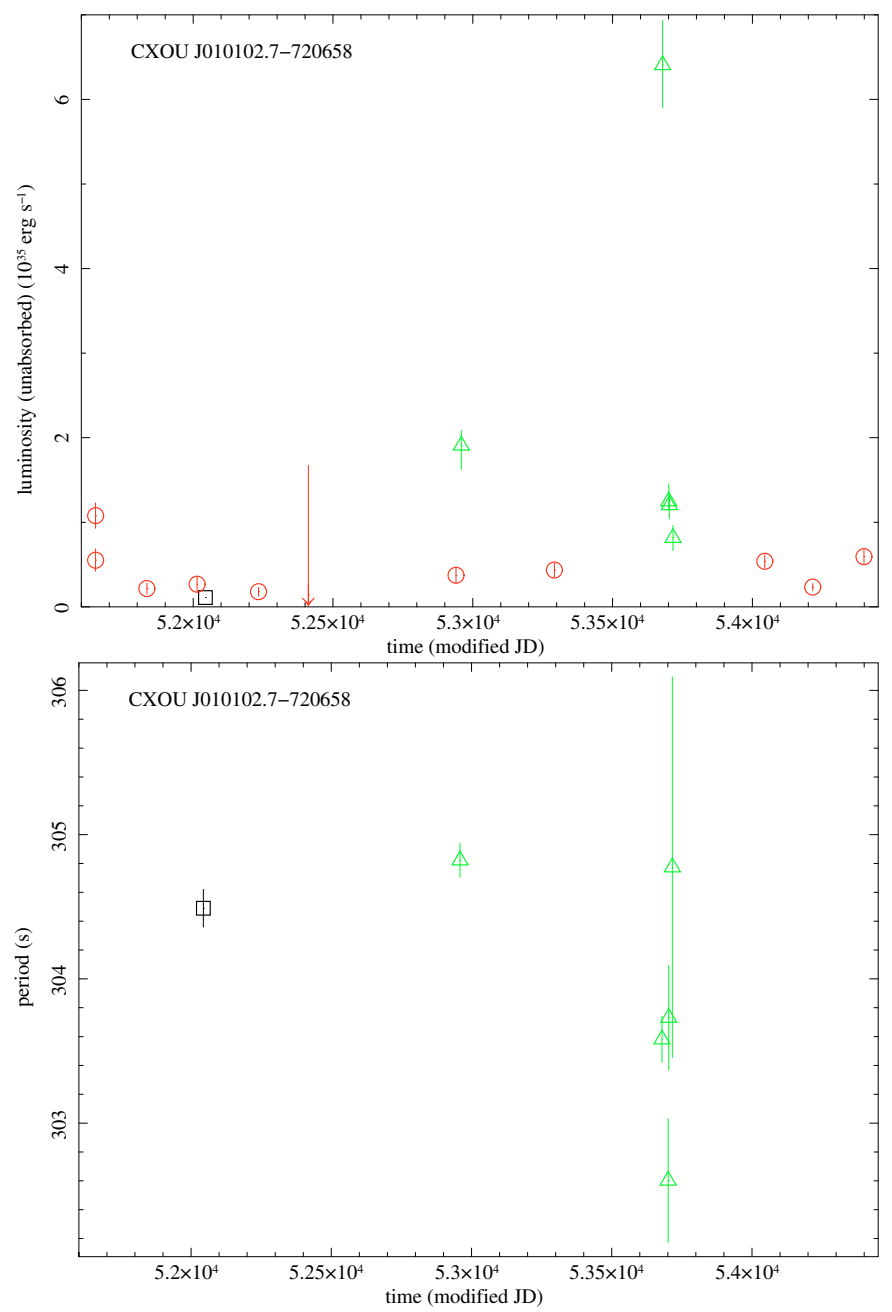

Fig. 5. Top: long term X-ray light-curve of CXOU J010102.7-720658 from this survey. Square (black): Macomb et al. (2003) (Chandra), circles (red): this work, luminosities from count-rate. Triangles (green): this work, luminosities from spectral fit. The source shows variability by a factor of $\sim 50$ in the X-ray luminosity. Bottom: long term pulse period history of CXOU J010102.7-720658. Square (black): Macomb et al. (2003) (Chandra), circles (green): this work.

powerlaw with photon indices larger than 1.6. Moreover, no $\mathrm{X}$-ray pulsations were found in any of these observations.

New results. RX J0101.6-7204 was observed twenty-six times with XMM-Newton and we performed spectral and temporal analyses on the EPIC data of all those detections. In agreement with the authors mentioned above, we measure photon indices around $1.9 \pm 0.3$ which is typical for background AGN and not for known Be/XRBs in the SMC. RX J0101.6-7204 shows a variability by factor of $\sim 3$ in the X-ray luminosity. Furthermore, no significant periodicity could be measured in any of the XMM-Newton detections.

The proposed optical counterpart of the ROSAT detection, [MA93] $1277=$ [MCPS] 3646296 (Meyssonnier \& Azzopardi 1993; Zaritsky et al. 2002), lies at distances between 3.4" to 8.1" from the XMM-Newton detections (see finding chart, Fig. 7). The relatively large scatter in the XMM-Newton positions, especially along the north-south direction, is probably caused by the large off-axis angle.
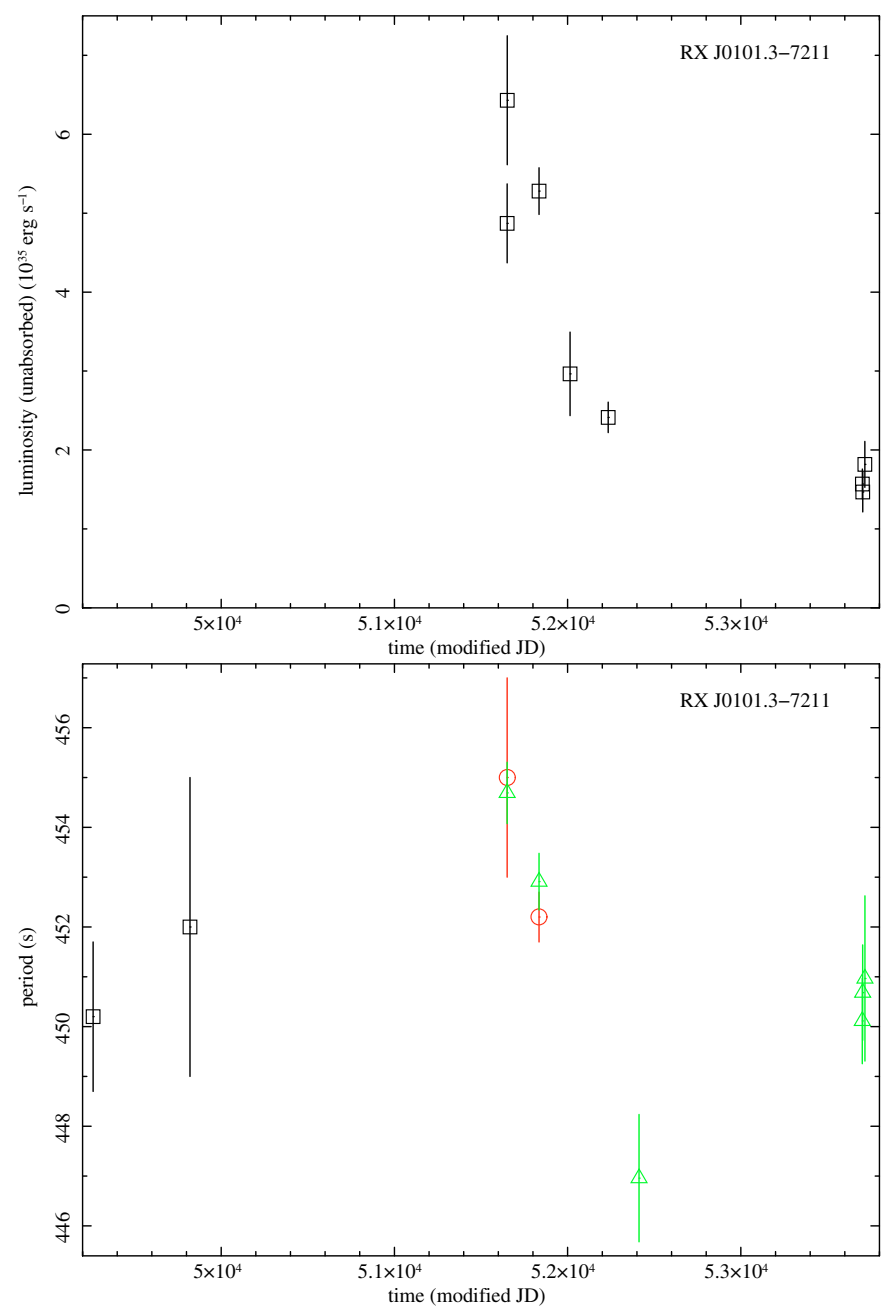

Fig. 6. Top: long term X-ray light-curve of RX J0101.3-7211 from this survey. Bottom: long term pulse period history of RX J0101.3-7211. Squares (black): Sasaki et al. (2001) (ROSAT), circles (red): Sasaki et al. (2001, 2003) (XMM), triangles (green): this work.



Fig. 7. DSS2 red finding chart of the region around RX J0101.6-7204. Solid circles (red): XMM-Newton detections, radii denote the statistical uncertainties, dashed circle (black): position of [MA93] $1277=$ [MCPS] 3646296. 

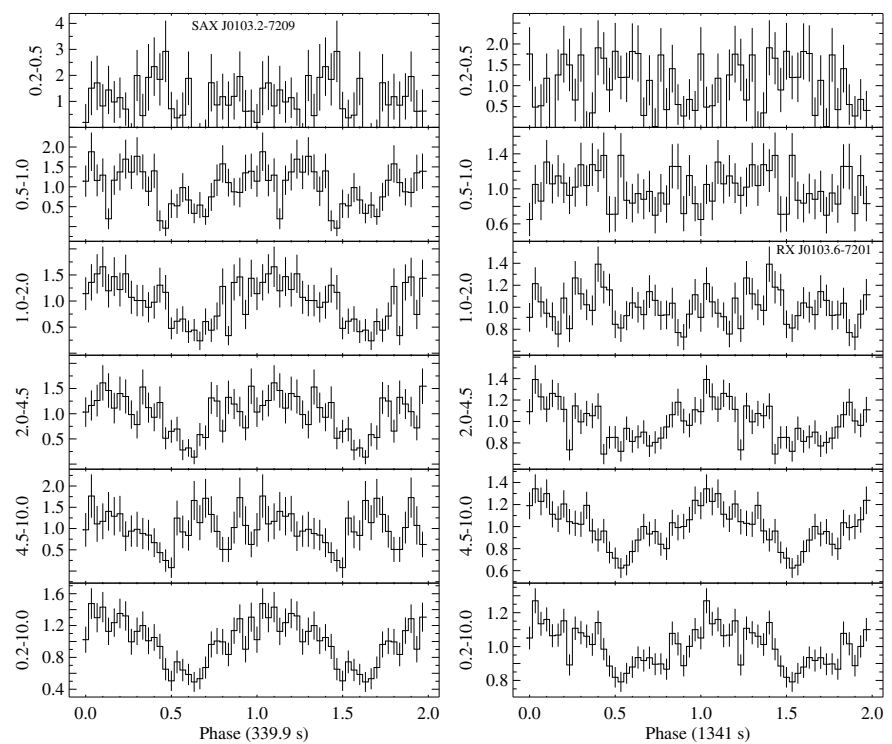

Fig. 8. Left: pulse profile of SAXJ0103.2-7209 from observation 0135722501, right: pulse profile of RX J0103.6-7201 from observation 0135722701 .

Based on our results, we conclude that RX J0101.6-7204 was wrongly identified with the emission line star [MA93] 1277, and is more likely a background AGN.

\subsection{The 345 s Be/X-ray binary pulsar SAX J0103.2-7209}

History. Israel et al. (1998) found $345.2 \pm 0.1 \mathrm{~s}$ pulsations with BeppoSAX in July 1998. By reanalyzing archival ASCA data from May 1996, Yokogawa \& Koyama (1998a) determined a period of $348.9 \pm 0.1$ s. Sasaki et al. (2003) and Haberl \& Pietsch (2004) were able to confirm the pulsations with XMM-Newton observations 0135720601 and 0110000301.

New results. Since the source is located in the same field as the calibration target 1E0102.2-7218, it has been observed twenty-two times with XMM-Newton so far. The long term X-ray light-curve (Fig. 9, top) shows a relatively constant brightness of $\sim 4 \times 10^{35} \mathrm{erg} \mathrm{s}^{-1}$ during most of the interval covered by XMM-Newton. However, SAX J0103.2-7209 was not detected in the last three observations which indicates a steep decline in the luminosity during the last part of the monitored period.

Furthermore, a linear pulsar spin-up is seen that ceased abruptly after May, 2002 (see Fig. 9, bottom). Because the X-ray light-curve does not show any sudden drop in luminosity at this time, a decrease in the accretion rate is probably not responsible for this change. More likely, either a reversal in the disc torque, as discussed by Bildsten et al. (1997) and Ghosh \& Lamb (1979), or the pulsar reaching its spin equilibrium might have caused the spin-up to stop.

The structure of the pulse profile shows no significant change during the covered period of 7.5 years. The modulation is dominated by a broad main pulse in all energy bands with narrow dips superimposed (see Fig. 8, left).

In contrast to the XMM-Newton observations, RXTE monitoring detects a large scatter in the pulsation period (Galache et al. 2008). This might be caused by RXTE detecting two different pulsars, SAX J0103.2-7209 and our newly discovered transient XMMU J005403.8-722632 (see Haberl et al. 2008a) with a similar period of $341.87 \mathrm{~s}$.

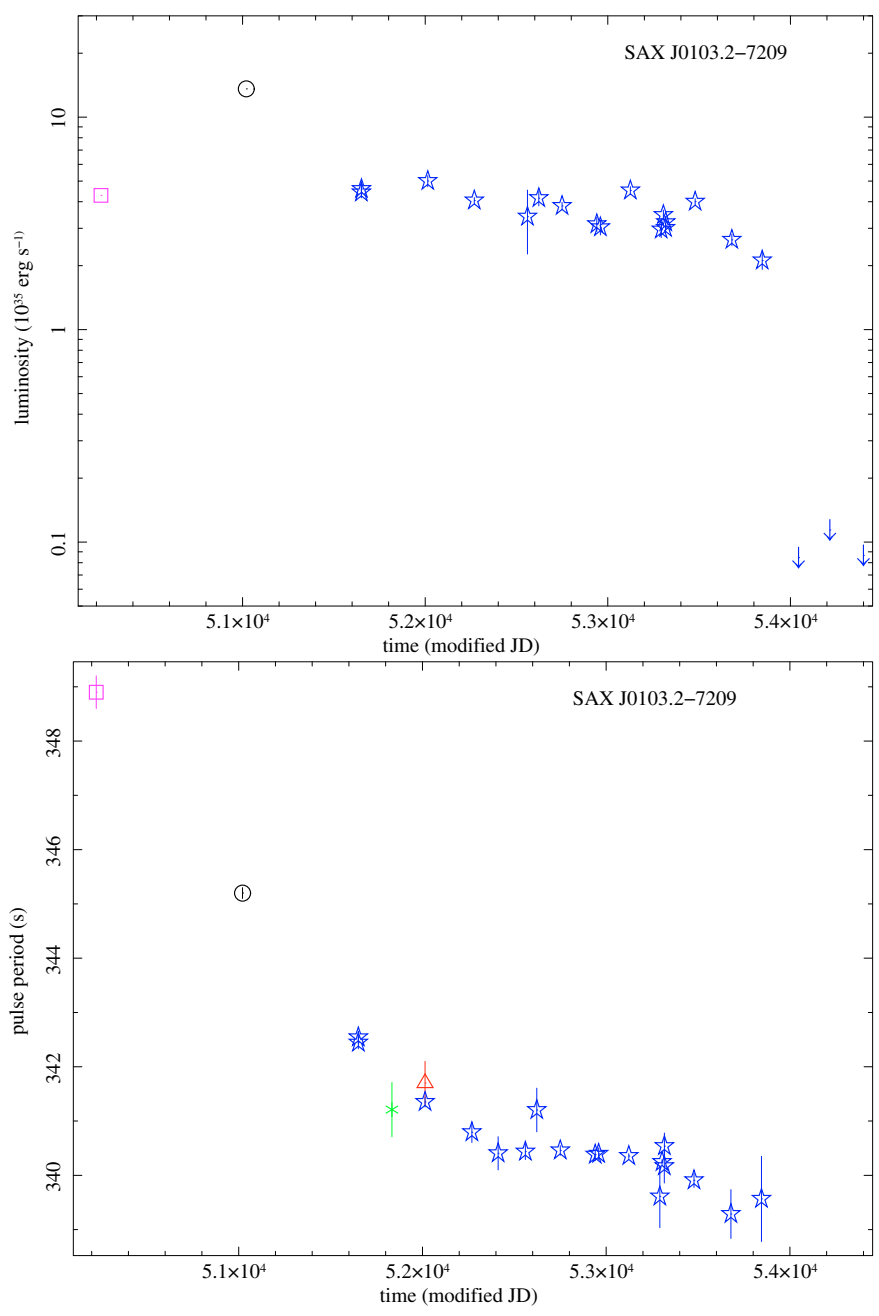

Fig. 9. Top: long term X-ray light-curve of SAX J0103.2-7209. Bottom: long term pulse period history of SAX J0103.2-7209. Square (magenta): Yokogawa \& Koyama (1998a) (ASCA), circle (black): Israel et al. (1998) (BeppoSAX), triangle (red): Sasaki et al. (2003) (XMM), cross (green): Haberl \& Pietsch (2004) (XMM), stars and upper limits (blue): this work.

The X-ray spectra can be modelled with an absorbed powerlaw. In some spectra a soft excess around $1 \mathrm{keV}$ appeared that could, however, not be modelled due to insufficient statistics. We therefore added all available spectra for each EPIC instrument and averaged the detector responses by weighting with the exposure time. A powerlaw fit with and without an additional thermal plasma emission component (mekal, best fit gives $k T=0.246 \pm 0.015 \mathrm{keV}, N_{\mathrm{H}}=(9.7 \pm 2.7) \times 10^{20} \mathrm{~cm}^{-2}$, $\gamma=0.78 \pm 0.02)$ are shown in Fig. 10 .

\subsection{The 1323 s Be/X-ray binary pulsar RX J0103.6-7201}

History. RX J0103.6-7201 is the pulsar with the longest period $(\sim 1323$ s) found in the SMC so far. Haberl \& Pietsch (2005) detected this source with XMM-Newton in nine observations between April 2000 and October 2004 and identified the emission line star [MA93] 1393 as the optical counterpart. They performed spectral and temporal analyses on the EPIC data of all those observations and detected the extremely long period four times. 

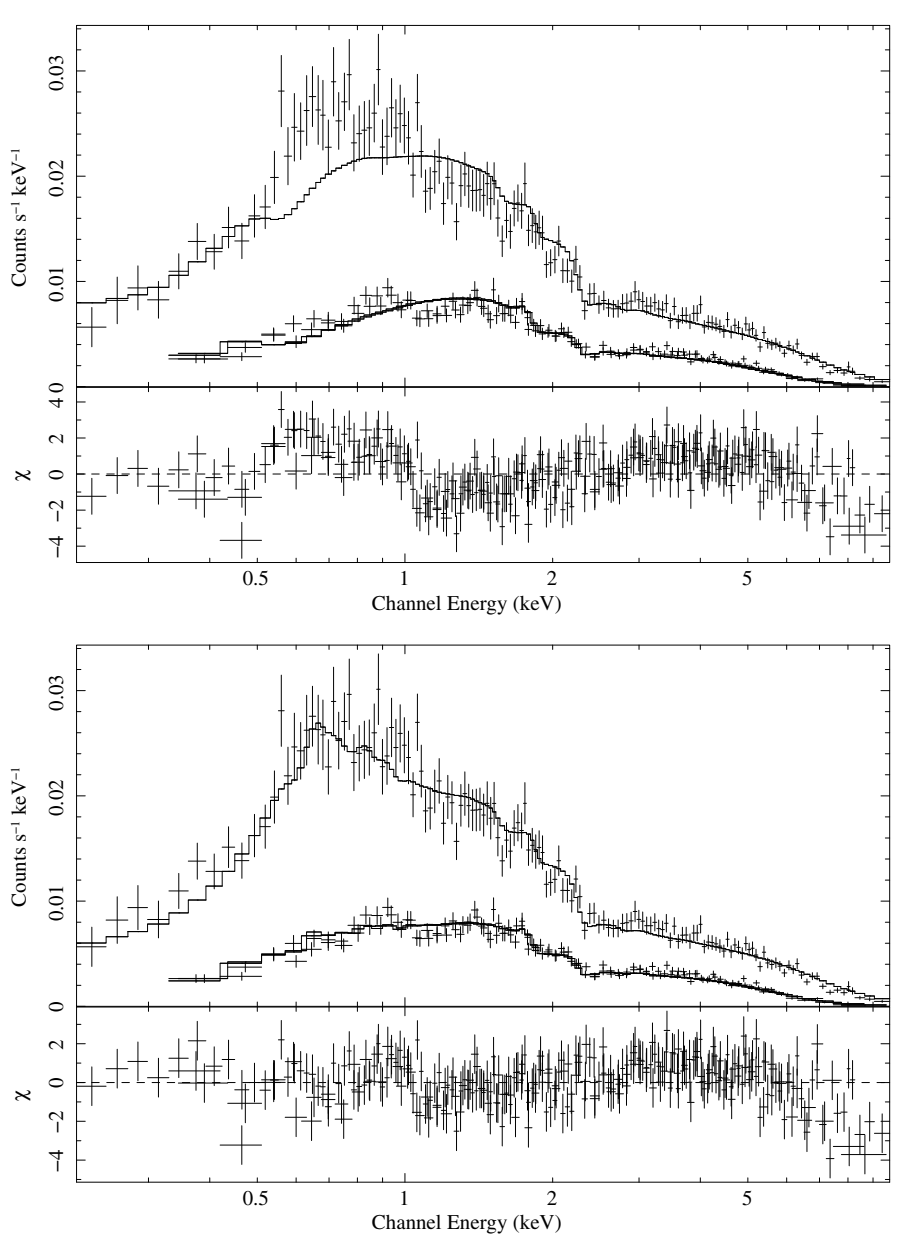

Fig. 10. PN, MOS1 and MOS2 sum spectra of SAX J0103.2-7209. Top: absorbed powerlaw. Bottom: absorbed powerlaw + mekal component $(k T=0.246 \pm 0.015 \mathrm{keV})$. The reduced $\chi^{2}$ improves from 1.8 to 1.3 after adding the mekal component.

The X-ray spectra were modelled with a hard powerlaw and a soft mekal component and show strong variation with time. Haberl \& Pietsch (2005) reported about one observation from October 2004 where the powerlaw component was strongly absorbed $\left(N_{\mathrm{H}}=1.07 \times 10^{23} \mathrm{~cm}^{-2}\right)$ and the soft component appeared as the dominant feature in the spectrum.

New results. Excluding a high background observation (0135722201), RXJ0103.6-7201 was observed twenty-one times so far with XMM-Newton, since it is also located in the same field as the calibration source 1E0102.2-7218. We reanalyzed previous data and added results from new observations. In sixteen cases the count-rate was sufficient for spectral modelling. In addition to the results from Haberl \& Pietsch (2005), the period was detected in four more observations with a rather large scatter from 1301 to $1340 \mathrm{~s}$ around a mean value of $\sim 1320 \mathrm{~s}$. As already mentioned by Haberl \& Pietsch (2005), the major uncertainty in the determination of this long period is likely caused by superimposed long term variations. This can lead to changes in the measured period larger than the statistical uncertainty might suggest. The pulse profiles show little modulation below $2 \mathrm{keV}$. At higher energies the pulse is dominated by a broad peak with an indication of a smaller interpulse (see Fig. 8, right).
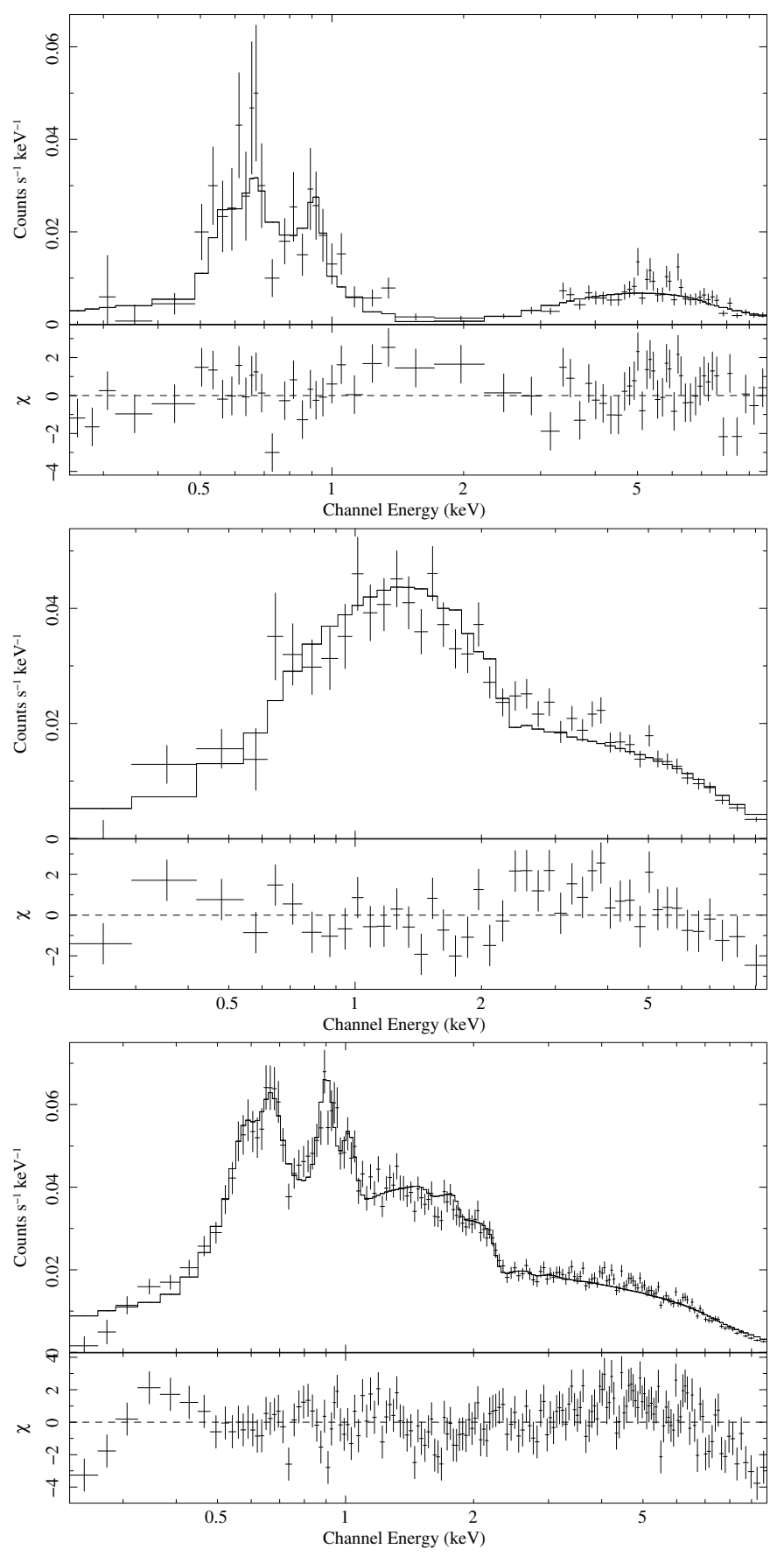

Fig. 11. Top: X-ray spectrum of RX J0103.6-7201 from October 14, 2004. The powerlaw component is highly absorbed $\left(N_{\mathrm{H}}=3.7 \times 10^{23} \mathrm{~cm}^{-2}\right)$ and the MEKAL component dominates the spectrum below $1 \mathrm{keV}\left(N_{\mathrm{H}}=1.1 \times 10^{22} \mathrm{~cm}^{-2}\right)$. Middle: X-ray spectrum of RXJ0103.6-7201 from October 26, 2007. The powerlaw is moderately absorbed $\left(N_{\mathrm{H}}=2.0 \times 10^{21} \mathrm{~cm}^{-2}\right)$ and the MEKAL component does not appear significantly. Bottom: fit using a model consisting of a powerlaw +4 Gaussian lines to the PN sum spectrum of RX J0103.6-7201 including all fourteen observations that showed soft excess.

A powerlaw + mekal model with a common absorption component was used to fit the spectra. However, it was necessary in three cases to treat the absorption of the two components separately (green circles in Fig. 12), because here only the powerlaw component was subject to high absorption whereas the mekal component was only moderately absorbed. Figure 11 shows two 


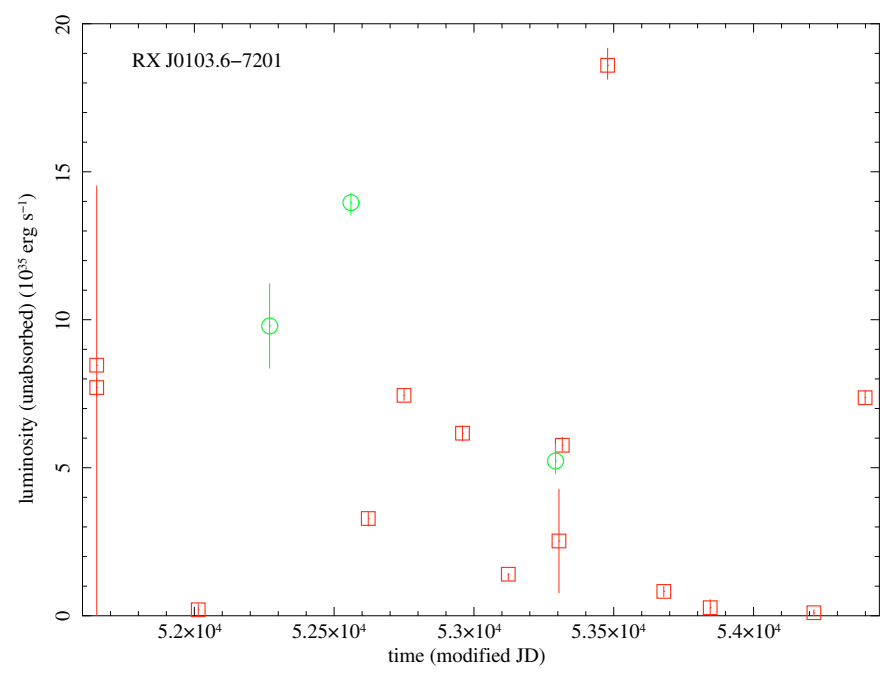

Fig. 12. Long term X-ray light-curve of RX J0103.6-7201, produced from powerlaw + mekal fits. Squares (red): common absorption for both components, circles (green): two separate absorptions.

extreme cases (top and middle) to illustrate the high spectral variability of this source. We note here that the values for $N_{\mathrm{H}}$ we derived are systematically higher than in Haberl \& Pietsch (2005) because we use elemental abundances reduced to 0.2 , as mentioned in Sect. 2.

To investigate the soft feature in more detail we added the PN source and background spectra and averaged the detector responses (weighted with exposure time) of all fourteen observations that showed an excess at $\sim 1 \mathrm{keV}$ (Fig. 11, bottom). In this way the contrast is greatly enhanced and the statistics are now sufficient to identify two line complexes at $0.56 \mathrm{keV}-0.66 \mathrm{keV}$ (O VII/VIII) and $0.9 \mathrm{keV}-1.3 \mathrm{keV}(\mathrm{Ne} \mathrm{IX} / \mathrm{X})$.

\section{9. $X M M U J 010509.7-721146=R X J 0105.1-7211$ is not the $3.34 \mathrm{~s}$ pulsar AX J0105-722}

History. Pulsations with a period of $3.34 \mathrm{~s}$ were detected with ASCA from AX J0105-722 by Yokogawa \& Koyama (1998b). The ASCA source could be resolved into several ROSAT sources by Filipović et al. (2000). The authors propose one of these objects, RX J0105.1-7211, as a Be/X-ray binary candidate, but they did not detect pulsations.

Haberl \& Pietsch (2004) detected an object with XMM-Newton within the error circle of both the ROSAT (RX J0105.1-7211) and ASCA detections, but no periodicity was found. Coe et al. (2005) analyzed the MACHO and OGLE data of the proposed optical counterpart of the ASCA source ([MA93] 1506) and discussed a possible orbital period of 11.09 days.

New results. We detected XMMU J010509.7-721146 from Haberl \& Pietsch (2004) in eighteen observations and identify it with the ROSAT source RX J0105.1-7211. Timing analyses of the X-ray data did not reveal any significant periodicity. The $\mathrm{X}$-ray spectra are well represented by an absorbed powerlaw model with a photon index of $2.0 \pm 0.3$, typical for background AGN.

Furthermore, there is no bright ( $V<18 \mathrm{mag}$ ) optical counterpart (from Zaritsky et al. 2002) within $9^{\prime \prime}$ radius. We therefore conclude that XMMUJ010509.7-721146 = RX J0105.1-7211 is not the ASCA pulsar AXJ0105-722, but more likely a background AGN. With XMM-Newton we do not find any other point source with a Be-like counterpart within the error circle of the ASCA detection.

\section{Summary}

We investigated Be/X-ray binaries in the north-eastern part of the SMC around the supernova remnant 1E0102.2-7218 which is observed by XMM-Newton as a calibration target every six months. The nearby X-ray binaries were monitored up to twentytwo times between April 2000 and October 2007 and we present their X-ray luminosity and pulse period history. Our investigated sources show very different variability factors with intensity variations of a factor of 3-4 (RX J0059.3-7223 and RX J0101.3-7211) to more than a factor of 100 (RX J0057.87207 and SAX J0102.3-7209) which is typical for Be/X-ray binaries (e.g. Haberl \& Pietsch 1999). Although some outbursts may have been missed due to the wide observational sampling, the range in variability likely reflects different binary separations, orbit eccentricities and Be disc sizes and densities (Okazaki \& Negueruela 2001).

The spin period histories of the investigated Be/X-ray binaries show episodes of spin-down and spin-up, the latter often associated with increased mass accretion during X-ray outbursts (e.g. CXOU J010102.7-720658 and RX J0101.3-7211). SAX J0103.2-7209 shows a different behaviour: After an apparently linear decrease in pulse period over about six years, the pulse period stayed nearly constant for about two years, before it again started to decrease. However, during the times when the period derivative changed, no significant change in X-ray luminosity was seen. During five years of monitoring of accretion powered pulsars in the Galaxy with BATSE, Bildsten et al. (1997) found a strong correlation of the accretion torque with pulsed flux during outbursts of transient pulsars, but no correlation or even anti-correlation in persistent (wind fed) sources. This could indicate that the neutron star in SAX J0103.2-7209 only temporarily accretes via a disc. The continued monitoring of this binary pulsar to follow its spin period after the recent drop in X-ray luminosity is important.

The X-ray spectra of two of our investigated sources show a soft spectral component dominated by emission lines of oxygen. Such emission from an optically thin plasma is expected from the reprocessing of the hard X-rays from the neutron star by circumstellar matter (Hickox et al. 2004). It is interesting that SAX J0103.2-7209 shows such an emission component which is also observed in wind-accreting supergiant HMXBs. On the other hand the large variability in X-ray luminosity is typical for a Be-type system. The existence of HMXBs that show properties of disc accreting (usually Be type) and wind accreting (supergiant systems) suggests a smooth transition between the two types of HMXBs.

Acknowledgements. The XMM-Newton project is supported by the Bundesministerium für Wirtschaft und Technologie/Deutsches Zentrum für Luft- und Raumfahrt (BMWI/DLR, FKZ 50 OX 0001) and the Max-Planck Society. The "Second Epoch Survey" of the southern sky was produced by the Anglo-Australian Observatory (AAO) using the UK Schmidt Telescope. Plates from this survey have been digitized and compressed by the ST ScI. Produced under Contract No. NAS 5-26555 with the National Aeronautics and Space Administration.

\section{References}

Bildsten, L., Chakrabarty, D., Chiu, J., et al. 1997, ApJS, 113, 367

Coe, M. J., Edge, W. R. T., Galache, J. L., \& McBride, V. A. 2005, MNRAS, 356,502 
Eger, P., \& Haberl, F. 2008, A\&A, 485, 807

Filipović, M. D., Haberl, F., Pietsch, W., \& Morgan, D. H. 2000, A\&A, 353, 129 Galache, J. L., Corbet, R. H. D., Coe, M. J., et al., 2008, ApJS, 177, 189

Ghosh, P., \& Lamb, F. K. 1979, ApJ, 234, 296

Gregory, P. C., \& Loredo, T. J. 1996, ApJ, 473, 1059

Haberl, F., \& Pietsch, W. 1999, A\&A, 344, 521

Haberl, F., \& Pietsch, W. 2004, A\&A, 414, 667

Haberl, F., \& Pietsch, W. 2005, A\&A, 438, 211

Haberl, F., \& Pietsch, W. 2007, A\&A, 476, 317

Haberl, F., \& Sasaki, M. 2000, A\&A, 359, 573

Haberl, F., Eger, P., \& Pietsch, W. 2008a, ArXiv e-prints, 806

Haberl, F., Eger, P., Pietsch, W., Corbet, R. H. D., \& Sasaki, M. 2008b, A\&A, 485,177

Hickox, R. C., Narayan, R., \& Kallman, T. R. 2004, ApJ, 614, 881

Hilditch, R. W., Howarth, I. D., \& Harries, T. J. 2005, MNRAS, 357, 304

Israel, G. L., Stella, L., Campana, S., et al. 1998, IAU Circ., 6999, 1

Kahabka, P., Pietsch, W., Filipović, M. D., \& Haberl, F. 1999, A\&AS, 136, 81
Macomb, D. J., Fox, D. W., Lamb, R. C., \& Prince, T. A. 2003, ApJ, 584, L79

Majid, W. A., Lamb, R. C., \& Macomb, D. J. 2004, ApJ, 609, 133

Meyssonnier, N., \& Azzopardi, M. 1993, A\&AS, 102, 451

Nazé, Y., Manfroid, J., Stevens, I. R., Corcoran, M. F., \& Flores, A. 2004, ApJ, 608, 208

Okazaki, A. T., \& Negueruela, I. 2001, A\&A, 377, 16

Russell, S. C., \& Dopita, M. A. 1992, ApJ, 384, 508

Sasaki, M., Haberl, F., Keller, S., \& Pietsch, W. 2001, A\&A, 369, L29

Sasaki, M., Pietsch, W., \& Haberl, F. 2003, A\&A, 403, 901

Strüder, L., Briel, U., Dennerl, K., et al. 2001, A\&A, 365, L18

Turner, M. J. L., Abbey, A., Arnaud, M., et al. 2001, A\&A, 365, L27

Wilms, J., Allen, A., \& McCray, R. 2000, ApJ, 542, 914

Yokogawa, J., \& Koyama, K. 1998a, IAU Circ., 7009, 3

Yokogawa, J., \& Koyama, K. 1998b, IAU Circ., 7028, 1

Zaritsky, D., Harris, J., Thompson, I. B., Grebel, E. K., \& Massey, P. 2002, AJ, 123,855

Zavlin, V. E., Pavlov, G. G., Sanwal, D., \& Trümper, J. 2000, ApJ, 540, L25 